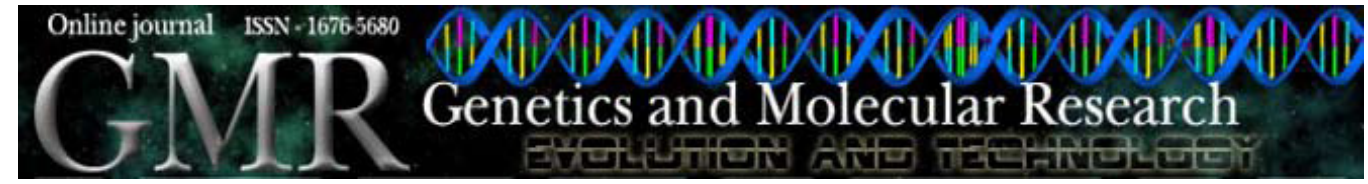

\title{
GSTT1, GSTM1, and GSTP1 polymorphisms and chemotherapy response in locally advanced breast cancer
}

\author{
A.L. Oliveira ${ }^{1}$, F.F.O. Rodrigues ${ }^{1}$, R.E. Santos $^{1}$, T. Aoki ${ }^{1}$, M.N. Rocha ${ }^{2}$, \\ C.A. Longui ${ }^{2}$ and M.B. Melo ${ }^{3}$ \\ ${ }^{1}$ Departamento de Ginecologia e Obstetrícia, \\ Irmandade da Santa Casa de Misericórdia de São Paulo, \\ Faculdade de Ciências Médicas, São Paulo, SP, Brasil \\ ${ }^{2}$ Laboratório de Medicina Molecular, Departamento de Fisiologia, \\ Irmandade da Santa Casa de Misericódia de São Paulo, \\ Faculdade de Ciências Médicas, São Paulo, SP, Brasil \\ ${ }^{3}$ Laboratório de Genética Molecular Humana, \\ Centro de Biologia Molecular e Engenharia Genética, \\ Universidade Estadual de Campinas, Campinas, SP, Brasil \\ Corresponding author: A.L. Oliveira \\ E-mail:drandrel@terra.com.br
}

Genet. Mol. Res. 9 (2): 1045-1053 (2010)

Received December 20, 2010

Accepted March 20, 2010

Published June 11, 2010

DOI 10.4238/vol9-2gmr726

\begin{abstract}
The glutathione S-transferase (GST) family consists of phase II detoxification enzymes that catalyze the conjugation of toxic substances, such as chemotherapeutic agents, to glutathione. We examined whether GSTT1/GSTT1"null", GSTM1/GSTM1"null" and GSTP1Ile105Ile/GSTP1Ile105Val polymorphisms are associated with different response rates to neoadjuvant chemotherapy in the treatment of stage II and III breast cancer. Forty Brazilian women with invasive ductal adenocarcinoma of the breast submitted to neoadjuvant chemotherapy, using 5-fluorouracil, epirubicin and cyclophosphamide, were genotyped for the GSTT1, GSTM1 and GSTP1 genes. Clinical
\end{abstract}


response was assessed by RECIST criteria. Comparisons were made for the three genes alone and in pairs, as polymorphic and as wildtype combinations and polymorphic/wild-type combinations. We analyzed all possible combinations and their response rate. Patients with the GSTT1/GSTP1105Ile combination were found to have a significantly better response than GSTT1"null"/GSTP1105Val (P $=0.0209)$ and GSTT1/GSTM1 $(\mathrm{P}=0.0376)$ combinations. Analysis of all possible combinations showed the GSTM1"null" polymorphic genotype to be present in four, and the wild-type GSTP1105Ile in six of the combinations associated with the largest number of responding patients. We found that patients with the GSTT1/GSTP1105Ile wild-type combination had a significantly higher response rate to chemotherapy than patients with the respective polymorphic GSTT1"null"/GSTP1105Val combination or patients with the wildtype GSTT1/GSTM1. The six gene combinations associated with the largest number of responding patients were found to contain the wildtype GSTP1105Ile and the polymorphic-type GSTM1"null". These specific combinations were virtually absent in the combinations with few responding patients.

Key words: Breast cancer; Polymorphism; Genetic; Drug therapy; Glutathione transferase

\section{INTRODUCTION}

Breast cancer is the most prevalent gynecological neoplasm with the highest incidence in women worldwide (Parkin et al., 2005). Late diagnosis of the disease and mechanisms of resistance to chemotherapeutic drugs are the greatest obstacles in treating breast cancer (Leonessa and Clarke, 2003). Different responses to similar chemotherapy schemes in breast cancer patients, having the same biologic characteristics and stage, suggest different mechanisms of resistance to this therapy, some of which are induced by genetic pathways (Hayes and Pulford, 1995; O'Brien and Tew, 1996; Pakunlu et al., 2003; Rodrigues et al., 2008). Molecular and biochemical aspects of the cellular resistance process have been described, where the genes of the glutathione S-transferase (GST) family have been shown to play an important role (Hayes and Pulford, 1995; O'Brien and Tew, 1996; Burg and Mulder, 2002; L'Ecuyer et al., 2004). These mechanisms of cellular resistance include metabolic detoxification by the GST family (Schisselbauer et al., 1990; Tew, 1994; Hayes and Pulford, 1995). The GSTP1, GSTM1 and GSTT1 genes, which belong to the GST family, encode the most important phase II detoxifying proteins involved in the conjugation of substrates that are toxic to cancer cells, including chemotherapeutic agents used in breast cancer treatment such as anthracyclines (Arrick and Nathan, 1984; Russo and Mitchell, 1985; Townsend and Cowan, 1989; Shea et al., 1990; Tew, 1994; Adler et al., 1999; Burg and Mulder, 2002; Townsend and Tew, 2003a,b; Daly, 2003; McIlwain et al., 2006). 
Of the different classes of genes found in the GST family, the GSTT1 form located on chromosome 22 is the most studied in the GSTT class, while the GSTM1 form located on chromosome 1 has been the most researched in the GSTM class. Both classes have polymorphic null forms (GSTT1"null" and GSTM1"null"), which do not have their two alleles and are therefore unable to encode the detoxifying enzymes (Cho et al., 2001; Townsend and Tew, 2003a,b; McIlwain et al., 2006). The GSTP class gene located on chromosome 11 has a wildtype form known as GSTP $1 * A$ (Ile105Ile/Ala113Ala) and two polymorphic forms known as $G S T P 1 * B$ (Ile105Val/Ala113Ala) and GSTP $1 * C$ (Ile105Val/Ala113Val), which, akin to the "null" forms, do not have their two alleles and therefore are unable to encode the detoxifying enzymes. The GSTM1"null" and GSTP1*B forms are also unable to inhibit their respective apoptosis pathways (ASK1 and JNK1) (Adler et al., 1999; Townsend and Tew, 2003a; Dang et al., 2005).

A number of different studies have yielded variable, inconsistent results regarding the relationship between the presence of polymorphic forms of GSTs and chemotherapeutic response (Riddick et al., 2005). Consequently, the aim of this study was to evaluate the clinical response to chemotherapy in patients with stage II and III invasive ductal adenocarcinoma of the breast in the presence of the GSTT1/GSTT1"null", GSTM1/GSTM1"null" and GSTP1Ile105Ile/GSTP1Ile105Val polymorphisms.

\section{MATERIAL AND METHODS}

\section{Patients and samples}

The study included 40 Brazilian patients diagnosed with stage II or III invasive ductal adenocarcinoma of the breast, who were consecutively referred to the chemotherapy outpatient unit of the Department of Obstetrics and Gynecology at Santa Casa de São Paulo Hospital, between February 2004 and December 2006. Inclusion criteria were as follows: i) a single, unilateral tumor without clinical or radiological signs of metastasis; ii) patient age between 35 and 70 years, and iii) absence of cardiomyopathy. Table 1 lists the patients and tumor characteristics.

The greater diameter of clinical margins of the patients' tumor was measured with pachymeter. It was tattooed before the first session of chemotherapy. Thirty days after the third session (just before surgery) we remeasured the major diameter using the same pachymeter and compared it to the tattooed initial margins. The clinical response to chemotherapy was evaluated according to RECIST criteria, adopted since 2002 for assessing chemotherapeutic response to solid tumor treatment. Individuals showing at least $30 \%$ tumor reduction are deemed responders and those showing less than 30\% tumor reduction are considered to be non-responders (Therasse et al., 2000).

Chemotherapy treatment consisted of three neoadjuvant administrations at 21-day intervals, following a 5-fluorouracil, epirubicin and cyclophosphamide scheme (FEC therapy).

\section{Multiplex polymerase chain reaction (PCR) and PCR-restriction fragment length polymorphism}

The genotypes of the GSTT1 and GSTM1 genes were determined by co-amplification 
Table 1. Demographic, clinical, pathologic, and genetic characteristics of the 40 patients enrolled in this study.

\begin{tabular}{|c|c|c|c|c|c|c|c|c|c|}
\hline Name & Race & IM & FM & CS & APM & CR & GSTT1 & GSTMI & GSTP1 \\
\hline ACFS & W & 7 & 5 & IIB & 6 & $\mathrm{Nr}$ & Wild & Wild & Ile105Val \\
\hline AMJP & W & 5 & 3 & IIA & 0.5 & $\operatorname{Pr}$ & Wild & "Null" & Ile105Val \\
\hline ALO & W & 4.5 & 2 & IIA & 1.5 & $\operatorname{Pr}$ & Wild & Wild & Ile105Ile \\
\hline BAC & W & 8 & 7 & IIIA & 3 & $\mathrm{Nr}$ & Wild & "Null" & Ile105Val \\
\hline CRN & B & 5 & 3 & IIIA & 4 & $\operatorname{Pr}$ & Wild & Wild & Ile105Ile \\
\hline DAA & W & 6 & 2 & IIB & 5 & $\operatorname{Pr}$ & "Null" & Wild & Ile105Ile \\
\hline DFM & B & 7 & 5.5 & IIIA & 8 & $\mathrm{Nr}$ & Wild & "Null" & Ile105Ile \\
\hline EL & W & 4 & 2 & IIB & 3 & $\operatorname{Pr}$ & "Null" & Wild & Ile105Val \\
\hline ERCM & B & 5 & 3.5 & IIB & 5.5 & $\operatorname{Pr}$ & Wild & "Null" & Ile105Ile \\
\hline EMFS & W & 4.5 & 2 & IIB & 2 & $\operatorname{Pr}$ & Wild & Wild & Ile $105 \mathrm{Val}$ \\
\hline ESN & B & 6 & 2 & IIIA & 3.5 & $\operatorname{Pr}$ & "Null" & Wild & Ile105Ile \\
\hline FGG & W & 4.5 & 3.5 & IIB & 5 & $\mathrm{Nr}$ & "Null" & Wild & Ile105Val \\
\hline GMS & W & 4 & 4 & IIB & 3 & $\mathrm{Nr}$ & "Null" & Wild & Ile105Ile \\
\hline GMS & B & 5.5 & 2 & IIIA & 4 & $\operatorname{Pr}$ & Wild & Wild & Ile105Ile \\
\hline IMF & B & 9 & 4.5 & IIIA & 6 & $\operatorname{Pr}$ & Wild & Wild & Ile105Val \\
\hline IAS & B & 8 & 6 & IIB & 9 & $\mathrm{Nr}$ & "Null" & "Null" & Ile105Ile \\
\hline JSS & B & 4 & 4 & IIB & 5 & $\mathrm{Nr}$ & Wild & "Null" & Ile105Val \\
\hline JP & W & 4 & 3.5 & IIA & 2.2 & $\mathrm{Nr}$ & Wild & Wild & Ile105Ile \\
\hline LSB & W & 8 & 6 & IIB & 6 & $\mathrm{Nr}$ & "Null" & "Null" & Ile105Ile \\
\hline MAEN & W & 10 & 8 & IIIA & 4 & $\mathrm{Nr}$ & Wild & Wild & Ile105Val \\
\hline MCRA & W & 5 & 2.5 & IIB & 2 & $\mathrm{Pr}$ & "Null" & Wild & Ile105Ile \\
\hline MAA & W & 8 & 7 & IIB & 4.5 & $\mathrm{Nr}$ & "Null" & "Null" & Ile105Ile \\
\hline MFG & W & 4 & 3 & IIA & 2 & $\mathrm{Nr}$ & Wild & Wild & Ile105Val \\
\hline MGS & B & 3 & 1.5 & IIB & 2 & $\mathrm{Pr}$ & Wild & Wild & Ile105Val \\
\hline NMP & W & 10 & 4.5 & IIB & 2 & $\operatorname{Pr}$ & "Null" & Wild & Ile105Val \\
\hline NOSS & W & 8 & 2 & IIIA & 4 & $\operatorname{Pr}$ & "Null" & Wild & Ile105Val \\
\hline OFS & B & 3.5 & 2 & IIB & 0.8 & $\operatorname{Pr}$ & Wild & Wild & Ile105Val \\
\hline $\mathrm{RR}$ & W & 4 & 3 & IIA & 0 & $\mathrm{Nr}$ & Wild & Wild & Ile105Val \\
\hline SM & B & 2.5 & 0 & IIB & 0 & $\mathrm{Cr}$ & "Null" & Wild & Ile105Ile \\
\hline TR & W & 4 & 2.5 & IIB & 3 & $\operatorname{Pr}$ & "Null" & Wild & Ile105Val \\
\hline ZLJ & W & 5.5 & 3 & IIIA & 0 & $\mathrm{Pr}$ & Wild & Wild & Ile105Ile \\
\hline MPA & B & 5 & 6 & IIB & 3.5 & $\mathrm{Nr}$ & "Null" & "Null" & Ile105Val \\
\hline MNA & W & 4 & 0 & IIB & 0 & $\mathrm{Cr}$ & "Null" & "Null" & Ile105Ile \\
\hline VOM & W & 6 & 0 & IIB & 0 & $\mathrm{Cr}$ & "Null" & Wild & Ile105Ile \\
\hline MJSA & W & 5 & 3 & IIB & 2.5 & $\operatorname{Pr}$ & Wild & "Null" & Ile105Val \\
\hline FPS & W & 6 & 7 & IIIA & 4.5 & $\mathrm{Nr}$ & Wild & Wild & Ile105Ile \\
\hline CMS & W & 2.5 & 1.5 & IIA & 2 & $\mathrm{Pr}$ & Wild & "Null" & Ile105Val \\
\hline EBSP & B & 8 & 6 & IIIA & 2 & $\mathrm{Nr}$ & "Null" & "Null" & Ile105Val \\
\hline DFS & B & 15 & 8.5 & IIIA & 7.5 & $\operatorname{Pr}$ & "Null" & "Null" & Ile105Val \\
\hline NCC & W & 4 & 2 & IIIA & 0.5 & $\operatorname{Pr}$ & Wild & Wild & Ile105Val \\
\hline Mean & $\mathrm{Na}$ & 5.77 & 3.37 & $\mathrm{Na}$ & 3.43 & $\mathrm{Na}$ & & & \\
\hline Median & $\mathrm{Na}$ & 5 & 3 & $\mathrm{Na}$ & 3 & $\mathrm{Na}$ & & & \\
\hline SD & $\mathrm{Na}$ & 2.45 & 1.86 & $\mathrm{Na}$ & 2.22 & $\mathrm{Na}$ & & & \\
\hline
\end{tabular}

$\mathrm{Nr}=$ no response $; \mathrm{Cr}=$ complete response; $\mathrm{Pr}=$ partial response; $\mathrm{Na}=$ not applicable. Correlation of 40 patients submitted to neoadjuvant chemotherapy by race ( $\mathrm{W}=$ Write; $\mathrm{B}=$ Black), initial clinical measurement of tumor (IM) and final clinical measurement of tumor (FM) in centimeters, clinical state (CS), anatomo-pathologic measurement (APM), clinical response (CR), and alleles identified.

using multiplex PCR, with the $\beta$-globin gene as an internal control, as described by Wilson et al. (2000). The primers used were 5'- CTT CCT TAC TGG TCC TCA CAT CTC -3' (sense) and 5'- TCA CCG GAT CAT GGC CAG CA -3' (anti-sense) for the GSTT1 gene, resulting in a 480-bp fragment; 5'- GAA CTC CCT GAA AAG CTA AAG C -3' (sense) and 5'- CTT GGG CTC AAA TAT ACG GTG G -3' (anti-sense) for the GSTM1 gene, resulting in a 215-bp fragment, and 5'-GAA GAG CCA AGG ACA GGT AC -3' (sense) and 5'- CAA CTT CAT CCA CGT TCA CC -3' (antisense) for the $\beta$-globin gene, resulting in a 268-bp fragment. The GSTT1"null" and GSTM1"null" variant forms were defined by the absence of the 480- and 215-bp fragments, respectively. 
GSTP1 gene-related products were obtained by PCR-restriction fragment length polymorphism, with 5'- ACC CCA GGG CTC TAT GGG AA -3' (sense) and 5'- TGA GGG CAC AAG AAG CCC CT -3' (anti-sense) primer, which generates a 176-bp product. The amplified product was then submitted to digestion with the Alw26I enzyme (New England Biolabs, Ipswich, MA, USA), yielding 91- and 86-bp fragments for the GSTP1105Val homozygous genotypes, 176-, 91- and 86-bp fragments for the GSTPIIle105Val heterozygous genotypes, and a 176-bp fragment for the wild-type GSTP1105Ile (Wilson et al., 2000).

\section{Statistical analysis}

The first part of the analysis consisted in comparing clinical response with any isolated gene (wild-type and polymorphic) and all possible combinations of genes in pairs (wild-type and/ or polymorphic). The hypothesis test was used, where the null hypothesis was when there is no association between clinical response (RECIST) and the isolated or combined gene, i.e., these variables are independent. Cramer correlations and a non-parametric test of the chi-square test were used for categorical variables. The maximum value of this test is 1 and the minimum is 0 . When the variables are independent, the value approaches 0. The data were input to the Statistical Program for the Social Sciences (SPSS, version 13.0, SPSS Inc., Chicago, IL, USA).

In the second part of the analysis, the test of equal proportions was used to analyze the variable clinical response (RECIST) between all possible combinations of genes in pairs (wild-type and/or polymorphic). We used the Microsoft Excel ${ }^{\circledR}$ software. $\mathrm{P}<0.05$ was considered to be statistically significant for both tests.

\section{RESULTS}

Among the 40 patients studied, $24(60 \%)$ were considered to be responders and $16(40 \%)$ nonresponders according to RECIST criteria. Twenty-two patients (55\%) showed the GSTT1 genotype, $18(45 \%)$ the GSTT1"null" genotype; 26 (65\%) the GSTM1 genotype, 14 (35\%) the GSTM1"null" genotype, $18(45 \%)$ the GSTP1 genotype, and $22(55 \%)$ the GSTPIIle105Val genotype.

Comparison of RECIST data for the genotype (individual genes) revealed no statistically significant difference in response between patients harboring either the polymorphic or the wild-type genotype (Table 2).

\begin{tabular}{|c|c|c|c|c|c|c|c|c|c|}
\hline \multirow[t]{2}{*}{ RECIST } & \multicolumn{2}{|c|}{ GSTT1 } & \multirow[t]{2}{*}{ P } & \multicolumn{2}{|c|}{ GSTM1 } & \multirow[t]{2}{*}{$\mathrm{P}$} & \multicolumn{2}{|c|}{ GSTP1 } & \multirow[t]{2}{*}{$\mathrm{P}$} \\
\hline & "Null" (\%) & Wild-type (\%) & & "Null" (\%) & Wild-type (\%) & & Ile105Val (\%) & Ile105Ile (\%) & \\
\hline $\mathrm{R}$ & $9(22.5)$ & $15(37.5)$ & 0.622172 & $11(27.5)$ & $13(32.5)$ & 0.068842 & $11(27.5)$ & $13(32.5)$ & 0.143526 \\
\hline NR & $9(22.5)$ & $7(17.5)$ & & $3(7.5)$ & $13(32.5)$ & & $11(27.5)$ & $5(12.5)$ & \\
\hline Total & 18 & 22 & & 14 & 26 & & 22 & 18 & \\
\hline
\end{tabular}

$\mathrm{R}=$ responders; $\mathrm{NR}=$ non-responders.

Comparison of data on the wild-type combinations against the respective polymorphictype combinations showed that eight patients $(100 \%)$ with the GSTT1/GSTP1105Ile combination responded to treatment as did four patients $(50 \%)$ with the GSTT1/GSTP1105Ile"null" combination, according to RECIST criteria $(\mathrm{P}=0.0209)$ (Table 3$)$. When wild-type geno- 
type combinations were compared with each other, and polymorphic genotype combinations were also compared with each other, according to the "responder" RECIST variable, nine patients $(60 \%)$ who had the GSTT1/GSTM1 combination and eight patients (100\%) who had the GSTT1/GSTP1105Ile combination were found to be responders $(\mathrm{P}=0.0376)$ (Table 4). Finally, two-by-two analysis of all possible combinations of genes according to the number of responders, showed the polymorphic-type GSTM1"null" to be present in four (66.6\%), and the wild-type GSTP1105Ile in three (50\%) of the six combinations associated with the largest number of responders. In addition, the polymorphic-type GSTM1"null" was not present in any of the six combinations in patients with poor response to chemotherapy, while the wild-type GSTP1105Ile was only present in one of these six combinations (17\%) (Table 5).

Table 3. Correlations between the numbers of responding patients according to the RECIST classification as to polymorphic- and their respective wild-type combinations of the GSTT1, GSTM1, and GSTP1 genes when combined in pairs.

\begin{tabular}{lcllrrr}
\hline Group 1 & AN & R & Group 2 & AN & R & P \\
\hline GSTT1"null"/GSTMI"null" & 7 & 5 & GSTTI/GSTMI & 15 & 9 & 0.6037 \\
GSTT1"null"/GSTPIIle105Val & 8 & 4 & GSTTI/GSTPIIle105Ile & 8 & 8 & 0.0209 \\
GSTMI"null"/GSTPIIle105Val & 8 & 7 & GSTM1/GSTPIIle105Ile & 12 & 9 & 0.4936 \\
\hline
\end{tabular}

$\mathrm{AN}=$ absolute number of cases; $\mathrm{R}=$ number of responders.

Table 4. Correlations between all possible polymorphic type combinations and between all possible wild-type combinations according to the number of responding patients based on the RECIST classification.

\begin{tabular}{|c|c|c|c|c|c|c|}
\hline Group 1 & $\mathrm{~N}$ & $\mathrm{R}(\%)$ & Group 2 & $\mathrm{~N}$ & $\mathrm{R}(\%)$ & $\mathrm{P}$ \\
\hline GSTT1"null”/GSTM1"null” & 7 & $5(71.4)$ & GSTT1"null”/GSTPIIle105Val & 8 & $4(50.0)$ & 0.3960 \\
\hline GSTT1/GSTM1 & 15 & $9(60.0)$ & GSTT1/GSTPIIle105Ile & 8 & $8(100.0)$ & 0.0376 \\
\hline GSTT1"null"/GSTPIIle105Val & 8 & $4(50.0)$ & GSTMI"null"/GSTPIIle105Val & 8 & $7(87.5)$ & 0.1052 \\
\hline GSTTI/GSTPIIle105Ile & 8 & $8(100.0)$ & GSTMI/GSTPIIle105Ile & 12 & $9(75.0)$ & 0.1260 \\
\hline GSTT1"null"/GSTMI"null" & 7 & $5(71.4)$ & GSTMI"null"/GSTPIIle105Val & 8 & $7(87.5)$ & 0.4412 \\
\hline GSTT1/GSTM1 & 15 & $9(60.0)$ & GSTMI/GSTPIIle105Ile & 12 & $9(75.0)$ & 0.4122 \\
\hline
\end{tabular}

$\mathrm{N}=$ number of cases; $\mathrm{R}=$ number of responders.

Table 5. Percentages of responders according to combinations of genes in pairs in decreasing order of frequency.

\begin{tabular}{|c|c|c|c|}
\hline Gene combination & $\mathrm{N}$ & $\mathrm{R}$ & $\%$ of responders \\
\hline GSTT1/GSTP1Ile105Ile & 8 & 8 & 100 \\
\hline GSTMI"null"/GSTPIIle105Val & 8 & 7 & 87.5 \\
\hline GSTT1/GSTM1"null" & 7 & 6 & 85.7 \\
\hline GSTMI/GSTPIIle105Ile & 12 & 9 & 75 \\
\hline GSTT1"null”/GSTM1"null” & 7 & 5 & 71.4 \\
\hline GSTMI"null"/GSTPIIle105Ile & 6 & 4 & 66.7 \\
\hline GSTT1/GSTM1 & 15 & 9 & 60 \\
\hline GSTT1/GSTPIIle105Val & 14 & 7 & 50 \\
\hline GSTT1"null"/GSTPIIle105Val & 8 & 4 & 50 \\
\hline GSTT1"null”/GSTP1Ile105ILe & 10 & 5 & 50 \\
\hline GSTM1/GSTT1"null" & 11 & 4 & 36.4 \\
\hline GSTM1/GSTP1Ile105Val & 14 & 4 & 28.6 \\
\hline
\end{tabular}

$\mathrm{N}=$ absolute number of patients observed for that combination; $\mathrm{R}=$ number of responders for that combination. 


\section{DISCUSSION}

Since the first evidence that glutathione S-transferases are involved in response to chemotherapy (Schisselbauer et al., 1990; Tew, 1994; Hayes and Pulford, 1995), the results of various subsequent studies have shown the inconsistent nature of this relationship (Riddick et al., 2005). In the present study, we investigated the possible association between polymorphisms in the GSTT1, GSTM1 and GSTP1 genes and response to chemotherapy in Brazilian women with stage II and III breast cancer submitted to neoadjuvant FEC therapy. When analyzed individually, none of the genes showed a statistically significant relationship with response to chemotherapy according to the Cramer test. Similar results were found by other authors (Moscow et al., 1989; Leyland-Jones et al., 1991; Alpert et al., 1997; Morrow et al., 1998; Allan et al., 2001; Yang et al., 2005). Lizard-Nacol et al., in 1999, studied 92 patients with breast cancer treated with neoadjuvant chemotherapy and did not find any association of clinical reduction of tumor size among patients with the wild-type or polymorphic form of GSTM1 genes. Yang et al., in 2005, studied 1602 women with breast cancer submitted to adjuvant chemotherapy and also did not observe a statistical difference in response between wild-type and polymorphic forms of the GSTM1 and GSTT1 genes. Allan et al., in 2001, studied the presence of acute myeloid leukemia in patients submitted to chemotherapy and did not observe a greater incidence of that disease when compared to patients with wild-type or polymorphic forms of the GSTT1 and GSTM1 genes.

However, when we combined the genes in pairs, the variant-type GSTMI"null" was present in four and the wild-type GSTP1105Ile in six of the combinations with the largest percentage of responders. Furthermore, in the six combinations with the lowest percentage of responders, the polymorphic-type GSTM1"null" was not present, while the wild-type GSTP1105Ile was present in only one combination. These results were also found by other authors when the GSTM1 gene was analyzed individually (Hamada et al., 1994; Morrow et al., 1998; O’Brien et al., 2000; Allan et al., 2001; Naoe et al., 2002; Huang et al., 2003; Yang et al., 2005), but were not replicated in the case of the GSTP1 gene. When the "responder" variable in the RECIST classification was used in comparing wild-type combinations with their respective variant polymorphic combinations, eight patients (100\%) with the GSTT1/GSTP1105Ile combination and four patients (50\%) with the GSTT1"null"/GSTP1105Val combination proved to be responders according to an analysis based on the test of equal proportions $(\mathrm{P}=0.0209)$. When the wild-type combinations were compared with each other, and the variant-type combinations were also compared with each other, nine patients $(60 \%)$ with the GSTT1/GSTM1 combination and eight patients $(100 \%)$ with the GSTT1/GSTP1105Ile combination proved to be responders based on the test of equal proportions $(\mathrm{P}=0.0376)$. These results suggest that the wild-type combination GSTT1/GSTP1105Ile was more sensitive to the chemotherapy used in this study. Our results are thus in contrast with those of other authors (Dirven, 1994; Tew, 1994; Howells et al., 2001; Paumi et al., 2001; Naoe et al., 2002; Khedhaier et al., 2003; Townsend and Tew, 2003a,b) who either found that the wild-types were more resistant than polymorphic forms or found no significant differences. Ambrosone et al., in 2001, conducted a retrospective study of breast cancer patients submitted to chemotherapy and noted greater recurrence-free survival among women who showed the polymorphic form of the GSTT1 and GSTM1 genes when compared to that with wild-type forms, thus in contrast with findings of other authors. Allan et al., in 2001, noted that acute myeloid leukemia was significantly more severe among patients who had the polymorphic 
form of the GSTP1 gene than patients with the wild-type form of the gene. Khedhaier et al., in 2003 , studied 309 breast cancer patients submitted to neoadjuvant chemotherapy and noted greater recurrence-free survival among patients who had the polymorphic form of the GSTT1 and GSTM1 genes, than patients with the wild form of genes. Yang et al., in 2005, studied 1602 breast cancer patients submitted to adjuvant chemotherapy and also noted greater recurrencefree survival among women with the polymorphic form of the GSTP1 gene.

As the wild-type genes are able to produce detoxifying enzymes that act in the metabolism of chemotherapeutic drugs and do take part in the inhibition of the JNK1 apoptosis pathway (Adler et al., 1999; Dang et al., 2005; McIlwain et al., 2006), our results were unexpected. Maybe a highly mixed racial origin represents a unique response to chemotherapy by Brazilian women, or other genetic factors related or not to the metabolism of drugs are involved. When interpreting these results, it should be borne in mind that this was a prospective study, and a larger sample is needed to guarantee statistical validity. Our results are drawn from an initial study, and further studies are warranted for confirmation.

\section{ACKNOWLEDGMENTS}

We are grateful to the Support Center for Scientific Publications of Santa Casa de São Paulo, Faculty of Medical Sciences, for editorial assistance.

\section{REFERENCES}

Adler V, Yin Z, Fuchs SY, Benezra M, et al. (1999). Regulation of JNK signaling by GSTp. EMBO J. 18: 1321-1334.

Allan JM, Wild CP, Rollinson S, Willett EV, et al. (2001). Polymorphism in glutathione S-transferase P1 is associated with susceptibility to chemotherapy-induced leukemia. Proc. Natl. Acad. Sci. U. S. A. 98: 11592-11597.

Alpert LC, Schecter RL, Berry DA, Melnychuk D, et al. (1997). Relation of glutathione S-transferase alpha and mu isoforms to response to therapy in human breast cancer. Clin. Cancer Res. 3: 661-667.

Ambrosone CB, Sweeney C, Coles BF, Thompson PA, et al. (2001). Polymorphisms in glutathione S-transferases (GSTM1 and GSTT1) and survival after treatment for breast cancer. Cancer Res. 61: 7130-7135.

Arrick BA and Nathan CF (1984). Glutathione metabolism as a determinant of therapeutic efficacy: a review. Cancer Res. 44: 4224-4232.

Burg D and Mulder GJ (2002). Glutathione conjugates and their synthetic derivatives as inhibitors of glutathione-dependent enzymes involved in cancer and drug resistance. Drug Metab. Rev. 34: 821-863.

Cho SG, Lee YH, Park HS, Ryoo K, et al. (2001). Glutathione S-transferase mu modulates the stress-activated signals by suppressing apoptosis signal-regulating kinase 1. J. Biol. Chem. 276: 12749-12755.

Daly AK (2003). Pharmacogenetics of the major polymorphic metabolizing enzymes. Fundam. Clin. Pharmacol. 17: $27-41$.

Dang DT, Chen F, Kohli M, Rago C, et al. (2005). Glutathione S-transferase pil promotes tumorigenicity in HCT116 human colon cancer cells. Cancer Res. 65: 9485-9494.

Dirven HA, van Ommen B and van Bladeren PJ (1994). Involvement of human glutathione S-transferase isoenzymes in the conjugation of cyclophosphamide metabolites with glutathione. Cancer Res. 54: 6215-6220.

Hamada S, Kamada M, Furumoto H, Hirao T, et al. (1994). Expression of glutathione S-transferase-pi in human ovarian cancer as an indicator of resistance to chemotherapy. Gynecol. Oncol. 52: 313-319.

Hayes JD and Pulford DJ (1995). The glutathione S-transferase supergene family: regulation of GST and the contribution of the isoenzymes to cancer chemoprotection and drug resistance. Crit. Rev. Biochem. Mol. Biol. 30: 445-600.

Howells RE, Holland T, Dhar KK, Redman CW, et al. (2001). Glutathione S-transferase GSTM1 and GSTT1 genotypes in ovarian cancer: association with p53 expression and survival. Int. J. Gynecol. Cancer 11: 107-112.

Huang J, Tan PH, Thiyagarajan J and Bay BH (2003). Prognostic significance of glutathione S-transferase-pi in invasive breast cancer. Mod. Pathol. 16: 558-565.

Khedhaier A, Remadi S, Corbex M, Ahmed SB, et al. (2003). Glutathione S-transferases (GSTT1 and GSTM1) gene deletions in Tunisians: susceptibility and prognostic implications in breast carcinoma. Br. J. Cancer 89: 1502-1507. 
L'Ecuyer T, Allebban Z, Thomas R and Vander Heide R (2004). Glutathione S-transferase overexpression protects against anthracycline-induced H9C2 cell death. Am. J. Physiol. Heart Circ. Physiol. 286: H2057-2064.

Leonessa F and Clarke R (2003). ATP binding cassette transporters and drug resistance in breast cancer. Endocr. Relat. Cancer 10: 43-73.

Leyland-Jones BR, Townsend AJ, Tu CP, Cowan KH, et al. (1991). Antineoplastic drug sensitivity of human MCF-7 breast cancer cells stably transfected with a human alpha class glutathione S-transferase gene. Cancer Res. 51: 587-594.

Lizard-Nacol S, Coudert B, Colosetti P, Riedinger JM, et al. (1999). Glutathione S-transferase M1 null genotype: lack of association with tumour characteristics and survival in advanced breast cancer. Breast Cancer Res. 1: 81-87.

McIlwain CC, Townsend DM and Tew KD (2006). Glutathione S-transferase polymorphisms: cancer incidence and therapy. Oncogene 25: 1639-1648.

Morrow CS, Smitherman PK, Diah SK, Schneider E, et al. (1998). Coordinated action of glutathione S-transferases (GSTs) and multidrug resistance protein 1 (MRP1) in antineoplastic drug detoxification. Mechanism of GST A1-1- and MRP1-associated resistance to chlorambucil in MCF7 breast carcinoma cells. J. Biol. Chem. 273: 20114-20120.

Moscow JA, Townsend AJ and Cowan KH (1989). Elevation of pi class glutathione S-transferase activity in human breast cancer cells by transfection of the GSTpi gene and its effect on sensitivity to toxins. Mol. Pharmacol. 36: 22-28.

Naoe T, Tagawa Y, Kiyoi H, Kodera Y, et al. (2002). Prognostic significance of the null genotype of glutathione S-transferase-T1 in patients with acute myeloid leukemia: increased early death after chemotherapy. Leukemia 16: 203-208.

O'Brien ML and Tew KD (1996). Glutathione and related enzymes in multidrug resistance. Eur. J. Cancer 32: 967-978.

O'Brien M, Kruh GD and Tew KD (2000). The influence of coordinate overexpression of glutathione phase II detoxification gene products on drug resistance. J. Pharmacol. Exp. Ther. 294: 480-487.

Pakunlu RI, Cook TJ and Minko T (2003). Simultaneous modulation of multidrug resistance and antiapoptotic cellular defense by MDR1 and BCL-2 targeted antisense oligonucleotides enhances the anticancer efficacy of doxorubicin. Pharm. Res. 20: 351-359.

Parkin DM, Bray F, Ferlay J and Pisani P (2005). Global cancer statistics, 2002. CA Cancer J. Clin. 55: 74-108.

Paumi CM, Ledford BG, Smitherman PK, Townsend AJ, et al. (2001). Role of multidrug resistance protein 1 (MRP1) and glutathione S-transferase A1-1 in alkylating agent resistance. Kinetics of glutathione conjugate formation and efflux govern differential cellular sensitivity to chlorambucil versus melphalan toxicity. J. Biol. Chem. 276: 7952-7956.

Riddick DS, Lee C, Ramji S, Chinje EC, et al. (2005). Cancer chemotherapy and drug metabolism. Drug Metab. Dispos. 33: 1083-1096.

Rodrigues FF, Santos RE, Melo MB, Silva MA, et al. (2008). Correlation of polymorphism C3435T of the MDR-1 gene and the response of primary chemotherapy in women with locally advanced breast cancer. Genet. Mol. Res. 7: 177-183.

Russo A and Mitchell JB (1985). Potentiation and protection of doxorubicin cytotoxicity by cellular glutathione modulation. Cancer Treat. Rep. 69: 1293-1296.

Schisselbauer JC, Silber R, Papadopoulos E, Abrams K, et al. (1990). Characterization of glutathione S-transferase expression in lymphocytes from chronic lymphocytic leukemia patients. Cancer Res. 50: 3562-3568.

Shea TC, Claflin G, Comstock KE, Sanderson BJ, et al. (1990). Glutathione transferase activity and isoenzyme composition in primary human breast cancers. Cancer Res. 50: 6848-6853.

Tew KD (1994). Glutathione-associated enzymes in anticancer drug resistance. Cancer Res. 54: 4313-4320.

Therasse P, Arbuck SG, Eisenhauer EA, Wanders J, et al. (2000). New guidelines to evaluate the response to treatment in solid tumors. European Organization for Research and Treatment of Cancer, National Cancer Institute of the United States, National Cancer Institute of Canada. J. Natl. Cancer Inst. 92: 205-216.

Townsend AJ and Cowan KH (1989). Glutathione S-transferases and antineoplastic drug resistance. Cancer Bull. 41: 31-36

Townsend D and Tew K (2003a). Cancer drugs, genetic variation and the glutathione-S-transferase gene family. Am. J. Pharmacogenomics 3: 157-172.

Townsend DM and Tew KD (2003b). The role of glutathione-S-transferase in anti-cancer drug resistance. Oncogene 22: 7369-7375.

Wilson MH, Grant PJ, Hardie LJ and Wild CP (2000). Glutathione S-transferase M1 null genotype is associated with a decreased risk of myocardial infarction. FASEB J. 14: 791-796.

Yang G, Shu XO, Ruan ZX, Cai QY, et al. (2005). Genetic polymorphisms in glutathione-S-transferase genes (GSTM1, GSTT1, GSTP1) and survival after chemotherapy for invasive breast carcinoma. Cancer 103: 52-58. 